\title{
AC Impedance Analysis for 10 Ah-Class Lithium-ion Batteries
}

\author{
Takeshi Kobayashi, ${ }^{\mathrm{a}, *}$ Shiro SeKI, ${ }^{\mathrm{a}}$ Yuichi Mita, ${ }^{\mathrm{a}}$ \\ Hajime Miyashiro, ${ }^{a}$ Nobuyuki TeradA, ${ }^{a}$ and Tooru KoJIMA ${ }^{b}$
}

\author{
${ }^{a}$ Central Research Institute of Electric Power Industry (2-11-1 Iwado Kita, Komae, Tokyo 201-8511, Japan) \\ ${ }^{b}$ Hitachi Vehicle Energy, Ltd. (2200 Oka, Fukaya, Saitama 369-0297, Japan)
}

Received November 30, 2009 ; Accepted January 19, 2010

\begin{abstract}
We measured impedance spectra for lithium-ion batteries with a large capacity over $10 \mathrm{Ah}$ and a small capacity under $1 \mathrm{Ah}$. We assigned the impedance spectra to the electrochemical parameters for the positive and negative electrode interfaces without disassembling. We proposed an advanced impedance analysis technique with constant phase element normalized by battery capacity for lithium-ion batteries of 10 Ah-class.
\end{abstract}

Key Words : AC Impedance, Constant Phase Element (CPE), Lithium-ion Battery, Activation Energy

\section{Introduction}

Lithium-ion batteries (LIBs) have attractive energy storage performance in terms of energy density, and power density.1) Much attention has been paid to LIBs to apply them to transportation applications such as hybrid electric vehicles (HEV) and electric vehicles (EV). ${ }^{2}$ Since LIBs with a longer calendar-life is required for such applications, ${ }^{3)}$ the techniques to evaluate the calendar-life will be of great importance.

Electrochemical impedance spectroscopy is a very helpful technique to separate the electrochemical reactions and to evaluate each parameter quantitatively. ${ }^{4}$ ) An ordinary method to analyze impedance data is to model the sample system by an appropriate equivalent circuit and compare the experimental data with the theoretical electrical response of the circuit. The LIB can be modeled by using an equivalent circuit consisting of some parallel connections of ohmic resistors and capacitors. The Nyquist diagram of an ideal battery such as a thinfilm battery gives precise semicircles.5) In most LIBs with large capacity, however, the data reveal a broadened semi-circle due to the complex electrochemical interfaces in the powder electrode systems. ${ }^{6}$ ) A common phenomenological approach is to describe a semicircle by the parallel connection of an ohmic resistor and a socalled 'constant phase element' (CPE); this connection is abbreviated to $R / / \mathrm{CPE}^{7)}$ This is because it's necessary to combine the some $R / / C$ for the analysis of the broadened semi-circle with the complex electrochemical reactions, and it's less simple to analysis its reactions. Since powder electrode materials are used in LIBs, the capacitor $C$ in this serial analog can be replaced with a $\mathrm{CPE}$ given by the following equation;

$$
Z(\mathrm{CPE})=1 / T(i \omega)^{P}
$$

where $i=(-1)^{0.5}, \omega=2 \pi f, f$ is frequency, $T$ is a CPE constant for capacitive dispersions, similar to $C$, whose unit is $\mathrm{Fs}^{P-1}$, and $P$ is a dimensionless value, $-1 \leq P \leq 1$. $^{7}$ These values will be a good indicator to analyze electrode reactions. Although the obtained values of the CPE are proportional to the reactive surface area, i.e. battery capacity, there has been little discussion to quantitatively refer them to battery capacity in previous reports.

In this study, we attempted to assign AC impedance spectra of LIBs with a large capacity over 10 Ah to evaluate the electrochemical reactions of the positive and negative electrode interfaces. Moreover, we studied the relationship between the battery capacities and impedance properties for LIBs with small and large capacities on the basis of the parameters of the CPE in the identical materials system.

\section{Experimental}

The equivalent circuit used in this study to describe the electrochemical reactions at the interface consists of an ohmic resistor $\mathrm{R}$ and a $\mathrm{CPE}$ connected in parallel. The total impedance is given by

$$
Z=(1 / R+1 / Z(\mathrm{CPE}))^{-1}
$$

Substituting of the equation (1) for CPE into equation (2) and splitting $Z$ into its real and imaginary parts yields,

$$
\begin{aligned}
& Z_{\text {im }}=-\left(R^{2} T \omega^{P} \sin (P \pi / 2)\right) / \\
& \left(1+2 R T \omega^{P} \cos (P \pi / 2)+\left(R T \omega^{P}\right)^{2}\right)
\end{aligned}
$$

The specific frequency is theoretically equal to the frequency at which $\mathrm{d} Z_{\text {im }} / \mathrm{d} \omega$ becomes zero. The specific frequency is calculated by differentiating Eq. (3) with respect to frequency, and substituting $\mathrm{d} Z_{\text {im }} / \mathrm{d} \omega=0$ in the differential equation. The numerator of the differential substituting $\mathrm{d} Z_{\text {im }} / \mathrm{d} \omega=0$ equation gives,

$$
P R^{2} T \omega^{P-1} \sin (P \pi / 2)\left(1-R^{2} T^{2} \omega^{2 P}\right)=0
$$

Therefore, the specific frequency is given by 


$$
f_{\text {top }}=(R T)^{-1 / P / 2 \pi}
$$

This equation was also confirmed using the equations, $C=R^{(1-P) / P} T^{1 / P}$ and $\omega R C=1{ }^{8)}$ The specific frequency is similar to the time constant, and it's useful as the indicator to compare the some reactions in the batteries.

The battery capacities of LIBs with small and large capacities were measured at $25^{\circ} \mathrm{C}$ using a Hokuto HJ0530 SM8 and a Biologic VMP3 connected to a $20 \mathrm{~A}$ booster. These batteries were discharged at a constant current of $1 \mathrm{CA}$ down to $2.7 \mathrm{~V}$ followed by charging at a constant current of $1 \mathrm{CA}$ up to $4.2 \mathrm{~V}$, then maintained potentiostatically at $4.2 \mathrm{~V}$ for $1.5 \mathrm{~h}$. The rest time between charge and discharge was $1 \mathrm{~h}$.

The impedance spectra were measured by a Biologic VMP3 at $15{ }^{\circ} \mathrm{C}$ at $10 \%$ SOC. The applied voltage was 5 $\mathrm{mV}$ with an applied frequency ranging from $10 \mathrm{kHz}$ to $50 \mathrm{mHz}$. The ZView of Scribners Associates Inc. was used as the impedance analysis program. ${ }^{9)}$

Apparent activation energies were calculated from the gradient in the Arrhenius plots of resistance values plotted against the inversed temperatures. An isothermal box was used for the measurement, set between $0{ }^{\circ} \mathrm{C}$ and $30{ }^{\circ} \mathrm{C}$ in every $5^{\circ} \mathrm{C}$ interval.

\section{Results and Discussion}

Table 1 shows the specifications of the LIBs with small and large capacities, in which the active material for the positive electrode was the same Ni-Mn-based material, while two types of active material were used for the negative electrode: hard carbon for [S-HC/NM] and graphite for $[\mathrm{S}-\mathrm{GR} / \mathrm{NM}]$ of the LIBs with small capacities. There are two types of cell shown in Table 1 : [S-HC/NM] of capacity around $290 \mathrm{mAh}$ and [S-GR/NM] of capacity around $370 \mathrm{mAh}$, the other of large capacity with $15 \mathrm{Ah}$ coded as [L-GR/NM]. There is no difference in electrode materials and electrolytic solution between [S-GR/NM] and [L-GR/NM]. The OCVs at $10 \%$ SOC for the three batteries proved to be $3.06 \mathrm{~V}$ for $[\mathrm{S}-\mathrm{HC} / \mathrm{NM}$ ], $3.50 \mathrm{~V}$ for [S-GR/NM], and 3.53 for [L-GR/NM].

Figure 1 shows the Nyquist diagrams (a) and the Bode diagrams (b) for $[\mathrm{S}-\mathrm{HC} / \mathrm{NM}]$ and $[\mathrm{S}-\mathrm{GR} / \mathrm{NM}]$ at $10 \%$ $\mathrm{SOC}$ and $15{ }^{\circ} \mathrm{C}$. The Nyquist diagram clearly reflects the multi step nature of lithium-ion insertion and extraction processes at the interfaces between the electrolytic solution and both electrodes in each LIB. Two semi-circles were assigned based on the Bode diagram in Fig. 1(b). The resistance and capacitance of each reaction component were calculated by least-squares fitting of the ac impedance data as reported by Tröltzsch et al. ${ }^{10)}$ The equivalent circuit consisted of two $R / / \mathrm{CPEs}$, in which one of the two resistances was connected in series with CPE3. The resistance of the bulk electrolytic solution, and the inductance of the cable in the system were connected to two $R / / \mathrm{CPEs}$. $R_{1} / / \mathrm{CPE} 1$ was assigned to the semi-circle of high frequency and $R_{2} / / \mathrm{CPE} 2$ to that of low frequency. CPE3 was also assigned to the diffusion component. The parameters obtained from fitting the impedance spectra measured for the batteries are summarized in Table 2. These parameters were calculated
Table 1 Specifications of the lithium-ion batteries.

\begin{tabular}{lccc}
\hline Battery type & [S-HC/NM] & [S-GR/NM] & {$[\mathrm{L}-\mathrm{GR} / \mathrm{NM}]$} \\
\hline Positive electrode & Ni-Mn based & Ni-Mn based & Ni-Mn based \\
Negative electrode & Hard carbon & Graphite & Graphite \\
Battery capacity & $368 \mathrm{mAh}$ & $291 \mathrm{mAh}$ & $14.8 \mathrm{Ah}$ \\
\hline
\end{tabular}
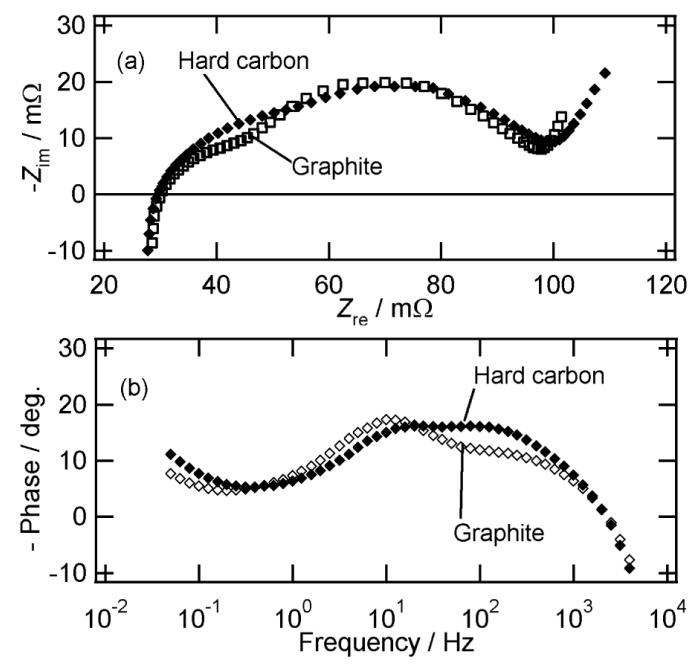

Fig. 1 Nyquist diagram (a), and Bode diagrams (b) for [S$\mathrm{HC} / \mathrm{NM}]$ and $[\mathrm{S}-\mathrm{GR} / \mathrm{NM}]$.

by fitting the data to the equivalent circuit.

It seems reasonable that the resistance and capacitance depended on the contact surface area between electrode and electrolytic solution, and the surface area is probably proportional to the amount of active electrode materials or the battery capacity. The resistance and capacitance should thus be normalized by the amount of active materials or the battery capacity. The parameters in Table 2 were normalized by the battery capacity, and the results are shown in Table 3. A $T$ of $\mathrm{CPE}$ was regarded as the similar capacitance at the interface between electrode and electrolytic solution. A normalized $T$ was calculated by dividing by battery capacity. $T_{1}$ in $[\mathrm{S}-\mathrm{HC} / \mathrm{NM}]$ for $\mathrm{CPE}$, normalized by the battery capacity, in the semi-circle of high frequency was three times as high as that in [S-GR/NM], whereas $P_{1}, T_{2}$ normalized by battery capacity and $P_{2}$ in semi-circle of low frequency were similar between [S-HC/NM] and $[\mathrm{S}-\mathrm{GR} / \mathrm{NM}]$.

The specific frequencies (SF) for each component were calculated by these parameters and Eq. (5). The SF for the semi-circles of high frequency in [S-HC/NM] was $180 \mathrm{~Hz}$, and the SF of low frequency was $11 \mathrm{~Hz}$. On the other hand, SF for the semi-circles of high frequency in [S-GR/NM] was $349 \mathrm{~Hz}$, the and the SF of low frequency was $7 \mathrm{~Hz}$. [S-HC/NM] and [S-GR/NM] showed similar $\mathrm{SF}$ for $R_{2} / / \mathrm{CPE} 2$, while they showed that of rather different $\mathrm{SF}$ for $R_{1} / / \mathrm{CPE} 1$.

These results suggest that $R_{1} / / \mathrm{CPE} 1$ of high frequencies relates to the reactions at negative electrode interface, and $R_{2} / / \mathrm{CPE} 2$ of low frequencies corresponds to that at the positive electrode interface.

Figure 2 shows the Nyquist diagram for [L-GR/NM] 
Table 2 Impedance parameters of the lithium-ion batteries.

\begin{tabular}{ccccccccccc}
\hline Battery type & $R_{0} / \mathrm{m} \Omega$ & $R_{1} / \mathrm{m} \Omega$ & $T_{1} / \mathrm{Fs}^{P-1}$ & $P_{1}$ & $R_{2} / \mathrm{m} \Omega$ & $T_{2} / \mathrm{Fs}^{P-1}$ & $P_{2}$ & $T_{3} / \mathrm{Fs}^{P-1}$ & $P_{3}$ & $\mathrm{Cap} . / \mathrm{Ah}$ \\
\hline [S-HC/NM] & 28 & 19 & 0.29 & 0.74 & 48 & 0.96 & 0.73 & $1.0 \mathrm{e} 2$ & 0.73 & 0.291 \\
[S-GR/NM] & 30 & 13 & 0.12 & 0.84 & 53 & 1.1 & 0.76 & $1.8 \mathrm{e} 2$ & 0.76 & 0.368 \\
[L-GR/NM] & 0.82 & 0.50 & 5.5 & 0.80 & 2.3 & 40 & 0.81 & $4.1 \mathrm{e} 3$ & 0.65 & 14.8 \\
\hline
\end{tabular}

Table 3 Impedance parameters normalized by battery capacities.

\begin{tabular}{cccccccccc}
\hline Battery type & $R_{0} / \mathrm{m} \Omega \mathrm{Ah}$ & $R_{1} / \mathrm{m} \Omega \mathrm{Ah}$ & $T_{1} / \mathrm{Fs}^{P-1}(\mathrm{Ah})^{-1}$ & $P_{1}$ & $R_{2} / \mathrm{m} \Omega \mathrm{Ah}$ & $T_{2} / \mathrm{Fs}^{P-1}(\mathrm{Ah})^{-1}$ & $P_{2}$ & $T_{3} / \mathrm{Fs} \mathrm{Fs}^{P-1}(\mathrm{Ah})^{-1}$ & $P_{3}$ \\
\hline [S-HC/NM] & 8.1 & 5.5 & 1.00 & 0.74 & 14 & 3.3 & 0.73 & 344 \\
[S-GR/NM] & 11 & 4.8 & 0.33 & 0.84 & 19 & 3.0 & 0.76 & 489 \\
[L-GR/NM] & 12 & 7.4 & 0.37 & 0.80 & 34 & 2.8 & 0.81 & 278 \\
\hline
\end{tabular}
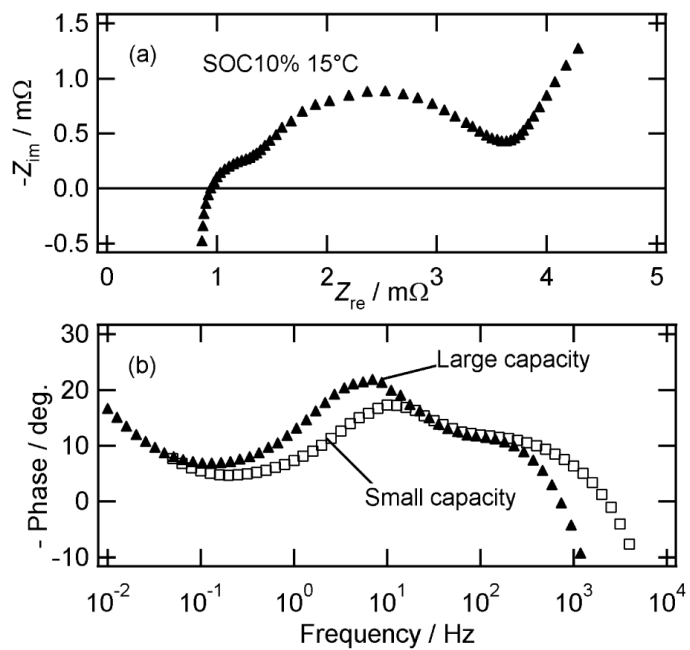

Fig. 2 Nyquist diagram for [L-GR/NM] (a), and Bode diagrams for [L-GR/NM] and [S-GR/NM] (b).

(a) and Bode diagrams (b) for [L-GR/NM] and [SGR/NM] at $10 \%$ SOC and $15{ }^{\circ} \mathrm{C}$. Two semi-circles were observed in Fig. 2(a) like Fig. 1(a). The parameters for the resistance and capacitance of each component in [L$\mathrm{GR} / \mathrm{NM}$ ] were obtained using the same equivalent circuit as that for batteries with small-capacities. The obtained parameters and their normalized values are also summarized in Tables 2 and 3. The normalized capacitances corresponding to the electrochemical reactions at the both electrode interfaces exhibited similar values for [S-GR/NM] and [L-GR/NM]. The SF for $R_{1} / / \mathrm{CPE} 1$ of [L-GR/NM] was $253 \mathrm{~Hz}$, and that for $R_{2} / /$ CPE2 was $3 \mathrm{~Hz}$. A significant difference of two orders of magnitude was confirmed between the values of SF for $R_{1} / / \mathrm{CPE} 1$ and $R_{2} / / \mathrm{CPE} 2$. These values for [L$\mathrm{GR} / \mathrm{NM}$ ] did not perfectly agree with those for [SGR/NM]. This would be caused by the different inductance components, which affect the SF for $R_{1} / / \mathrm{CPE} 1$, used in the battery configurations.

These results convinced that the assignment for $R_{1} / / \mathrm{CPE} 1$ and $R_{2} / / \mathrm{CPE} 2$ in [L-GR/NM] must be the same as in [S-GR/NM]. The SF and normalized $T$ of CPE were indicators to compare some batteries in the identical materials system.

Figure 3 shows the dependence of resistance on

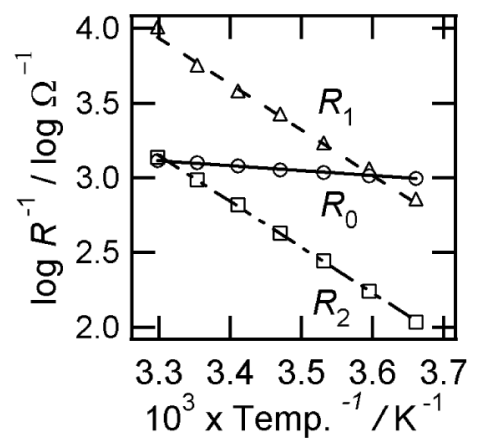

Fig. 3 Arrhenius plot for the electrochemical reactions corresponding to $R_{\mathrm{n}}(\mathrm{n}: 0-2)$ in [L-GR/NM].

inversed temperature in [L-GR/NM]. The apparent activation energies $\left(E_{a}\right)$ for the electrochemical reactions corresponding to $R_{\mathrm{n}}$ (n: $0-2$ ) were calculated from the gradients of the Arrhenius plots in Fig. 3. The $E_{a}$ of $R_{0}$, corresponding to the ionic conduction in electrolytic solution, was $7.0 \pm 0.2 \mathrm{~kJ} \mathrm{~mol}^{-1}$. ${ }^{11)}$ The $E_{a}$ of $R_{1}$ was $59 \pm 2 \mathrm{~kJ} \mathrm{~mol}^{-1}$, and that of $R_{2}$ was $59 \pm 1 \mathrm{~kJ} \mathrm{~mol}^{-1}$. There was no difference observed between these apparent activation energies for the both electrode interfaces.

\section{Conclusion}

We proposed an advanced method of impedance analysis for LIBs with a large capacity using resistance and capacitance normalized by the battery capacity. We assigned the semi-circles of the impedance spectra for the LIBs without disassembling, determining constant phase elements and the specific frequencies for both electrode interface reactions. We demonstrated that the impedance parameters depend on the battery capacity and found that the $T$ in the CPE normalized by battery capacity is an important indicator in ac impedance analysis and the battery performances.

\section{Acknowledgements}

This work was supported by New Energy and Industrial Technology Development Organization (NEDO) of Japan.

\section{References}

1) T. Kojima, T. Ishizu, T. Horiba, and M. Yoshikawa, J. 
Power Sources, 189, 859 (2009).

2) T. Horiba, T. Maeshima, T. Matsumura, M. Koseki, J. Arai, and Y. Muranaka, J. Power Sources, 146, 107 (2005).

3) I. Bloom, S. A. Jones, V. S. Battaglia, G. L. Henriksen, J. P. Christophersen, R. B. Wright, C. D. Ho, J. R. Belt, and C. G. Motloch, J. Power Sources, 124, 538 (2003).

4) M. Itagaki, S. Yotsuda, N. Kobari, K. Watanabe, S. Kinoshita, and M. Ue, Electrochim. Acta, 51, 1629 (2006).

5) I. Yamada, Y. Iriyama, T. Abe, and Z. Ogumi, J. Power Sources, 172, 933 (2007).

6) T. Osaka, S. Nakade, M. Rajamäki, and T. Momma, J. Power Sources, 119-121, 929 (2003).
7) J. R. Macdonald, Impedance spectroscopy, Wiley, New York, 1987, p. 27-128.

8) M. S. Abouzari, F. Berkemeier, G. Schmitz, and D. Wilmer, Solid State Ionics, 180, 922 (2009).

9) ZPLOT and ZVIEW for Windows, Scribner Associates Inc., North Carolina, USA.

10) U. Tröltzsch, O. Kanoun, and H. Tränkler, Electrochim. Acta, 51, 1664 (2006).

11) L. F. Li, H. S. Lee, H. Li, X. Q. Yang, K. W. Nam, W. S. Yoon, J. McBreen, and X. J. Huang, J. Power Sources, 184, 517 (2008). 